\title{
Erratum to: LPS Pretreatment Provides Neuroprotective Roles in Rats with Subarachnoid Hemorrhage by Downregulating MMP9 and Caspase3 Associated with TLR4 Signaling Activation
}

\author{
Ting-Hua Wang ${ }^{1} \cdot$ Liu-Lin Xiong ${ }^{1} \cdot$ Shuai-Fen Yang $^{1}$ - Chao You ${ }^{1}$ Qing-Jie Xia ${ }^{1}$. \\ Yang $\mathrm{Xu}^{1} \cdot$ Piao Zhang ${ }^{2} \cdot$ Shu-Fen Wang ${ }^{3} \cdot \mathrm{Jia} \mathrm{Liu}^{1,2}$
}

Published online: 6 December 2016

(C) Springer Science+Business Media New York 2016

\section{Erratum to: Mol Neurobiol}

DOI: 10.1007/s12035-016-0259-7

The original version of this article unfortunately contained a mistake.

The author's name Shu-Feng Wang should be changed to Shu-Fen Wang as presented above. The error appeared from the original manuscript. The authors hereby publish this correction note.

The original article was corrected.

The online version of the original article can be found under at http:// dx.doi.org/10.1007/s12035-016-0259-7.

\footnotetext{
Shu-Fen Wang wsf66@hotmail.com

liujiaaixuexi@126.com

Ting-Hua Wang

tinghua_neuron@263.net

1 Institute of Neurological Disease, and Department of Neurosurgery, Translational Neuroscience Center, the State Key Laboratory of Biotherapy, West China Hospital, Sichuan University,

Chengdu 610041, People's Republic of China

2 Animal Zoology Department, Institute of Neuroscience, Kunming Medical University, Kunming 650000, China

3 Yunnan Key Laboratory of Stem Cells and Regenerative Medicine, Institute of Molecular and Clinical Medicine, Kunming Medical University, Kunming 650000, China
}

$\triangle$ Jia Liu 